\title{
Near midplane scintillator-based fast ion loss detector on DIII-D ${ }^{a)}$
}

\author{
X. Chen, ${ }^{1, b)}$ R. K. Fisher, ${ }^{2}$ D. C. Pace,${ }^{2}$ M. García-Muñoz, ${ }^{3}$ J. A. Chavez, ${ }^{2}$ \\ W. W. Heidbrink, ${ }^{1}$ and M. A. Van Zeeland ${ }^{2}$ \\ ${ }^{1}$ University of California-Irvine, Irvine, California, USA \\ ${ }^{2}$ General Atomics, PO Box 85608, San Diego, California 92186-5608, USA \\ ${ }^{3}$ Max-Planck Institut fur Plasmaphysiks, Garching, Germany
}

(Presented 8 May 2012; received 4 May 2012; accepted 1 June 2012; published online 3 July 2012)

\begin{abstract}
A new scintillator-based fast-ion loss detector (FILD) installed near the outer midplane of the plasma has been commissioned on DIII-D. This detector successfully measures coherent fast ion losses produced by fast-ion driven instabilities $(\leq 500 \mathrm{kHz})$. Combined with the first FILD at $\sim 45^{\circ}$ below the outer midplane [R. K. Fisher, et al., Rev. Sci. Instrum. 81, 10D307 (2010)], the two-detector system measures poloidal variation of losses. The phase space sensitivity of the new detector (gyroradius $\mathrm{r}_{L}$ $\sim[1.5-8] \mathrm{cm}$ and pitch angle $\left.\alpha \sim\left[35^{\circ}-85^{\circ}\right]\right)$ is calibrated using neutral beam first orbit loss measurements. Since fast ion losses are localized poloidally, having two FILDs at different poloidal locations allows for the study of losses over a wider range of plasma shapes and types of loss orbits. (C) 2012 American Institute of Physics. [http://dx.doi.org/10.1063/1.4732063]
\end{abstract}

\section{INTRODUCTION}

In fusion plasmas, fast ions can be generated by fusion reactions, neutral-beam injection, and radio-frequency heating. These fast ions must be well-confined until their energy is transferred to the bulk plasma. Losses of fast ions can potentially damage the first wall and deteriorate the heating and current drive efficiency. Understanding the loss mechanisms is important for predicting the performance of future fusion reactors.

Fast ions born on open orbits connected with the first wall are described as first orbit losses or prompt losses. In addition to prompt loss, fast ion losses can be induced by the magnetic background directly, e.g., ripple losses, test blanket modules (TBMs) and resonant magnetic perturbations (RMPs), and by magnetohydrodynamic (MHD) perturbations. These perturbations, including Alfvén instabilities destabilized by fast ions and low-frequency MHD modes from bulk plasma, and their associated fast-ion losses are routinely observed in present devices. ${ }^{1,2}$

Fast ion losses can be measured by many different types of diagnostics. On DIII-D there are Faraday collector-based beam ion loss detectors (BILD), ${ }^{3}$ scintillator-based fast-ion loss detectors (FILD) described in this paper, and a number of less direct loss diagnostics. ${ }^{4}$ With no midplane ports available at the time, the first FILD on DIII-D was installed $\sim 45^{\circ}$ below the outer midplane in 2010 (Ref. 5) and has successfully measured prompt losses and coherent losses produced by fast-ion driven instabilities. ${ }^{6}$ In the 2011 campaign, a second FILD detector was installed near the outer midplane $\left(\Theta_{\text {poloidal }}\right.$ $\approx 0^{\circ}$ ) (Fig. 1), which is the optimal detection location for many loss processes. ${ }^{7,8}$

\footnotetext{
a) Contributed paper, published as part of the Proceedings of the 19th Topical Conference on High-Temperature Plasma Diagnostics, Monterey, California, May 2012.

b)Electronic mail: chenxi@fusion.gat.com.
}

This paper is organized as follows. A detailed description of the fast ion loss detectors, especially the newly installed midplane FILD, is given in Sec. II. Some experimental results are reported and discussed in Sec. III.

\section{FAST-ION LOSS DETECTOR (FILD)}

Fast ions on their gyro-orbits pass through a collimating aperture and impinge on a scintillator, inducing light. The newly installed midplane FILD (FILD2) uses the same kind of scintillator (Lightscape Materials, Maui535, formerly TGGreen) as the previous FILD (FILD1). A schematic of the FILD2 system is shown in Fig. 1. A 50/50 beam splitter divides the scintillator light into two branches. One branch is imaged onto a charge-coupled device (CCD) camera with a Schneider-Kreuznach Xenon ( $25 \mathrm{~mm}$ f/0.95) lens. This PCO Pixelfly VGA 12-bit CCD camera is controlled from a PCI board installed in a dedicated PC and records the light signals throughout the discharge with a maximum 160 frames per second (fps) sampling rate. The incident fast ions with different energies and pitch angles disperse onto different spots on the scintillator plate. The higher the energy (the larger the gyroradius), the further the striking spot is away from the aperture. The larger the velocity parallel to local magnetic field at the aperture, the smaller the pitch angle of the ion.

In this paper, pitch angle is defined as $\left[\alpha=\cos ^{-1}\left(\mathrm{v}_{\|} / \mathrm{v}\right)\right]$ where zero means traveling parallel to the total magnetic field direction at the aperture. Therefore the two-dimensional light distribution on the scintillator contains information about the energy and pitch angle of lost ions. The other branch of scintillator light after the beam splitter is collected by a rectangular bundle of 20 Fibergude SPI1500/1650N plastic clad silica fibers ( $1500 \mu \mathrm{m}$ diameter, $45 \mathrm{~m}$ long). Each of the 5 fiber by 4 fiber array views a different region of gyroradius and pitch angle on the scintillator. The light from each fiber is then coupled to a Hamamatsu H10721 photomultiplier tube (PMT). The PMT output current (typical value 5-7 $\mu \mathrm{A}$ ) is amplified 


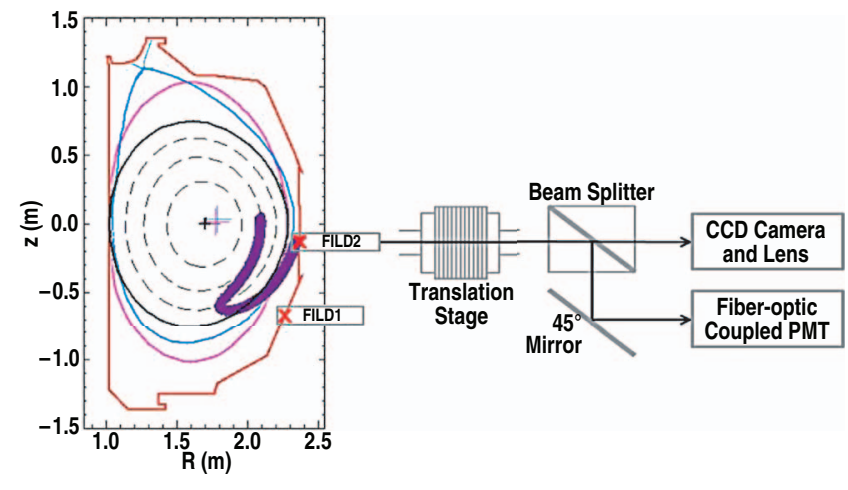

FIG. 1. Poloidal locations of FILD1 ( $\sim 5^{\circ}$ below midplane) and midplane FILD2 (schematic of the FILD2 system). Three types of plasma shapes are shown: oval (magenta), divertor (blue), and circular (black). Three flux surfaces (black dash) and one calculated ion orbit (violet), which originated from the 330L neutral beam and is detected at FILD2 but not FILD1, are also shown for a circular plasma case (shot 146 103, $494 \mathrm{~ms}$ ).

at a gain of $0.1 \mathrm{~V} / \mu \mathrm{A}$ and digitized at $1 \mathrm{MHz}$. The fast time response of the PMT is sufficient to track fast ion loss induced by high-frequency Alfvén eigenmodes.

FILD1 is located at $\sim 45^{\circ}$ below the midplane and $225^{\circ}$ toroidal angle; FILD2 is located $\sim 15 \mathrm{~cm}$ below the midplane and $165^{\circ}$ toroidal angle, as shown in Fig. 1. Both FILDs are radially insertable through vacuum translation stages (MDC Vacuum Products LMT-254) to achieve the optimal compromise between high signal level and low plasma and beam ion heat loads. FILD1 is usually inserted with the aperture well past the front face of the plasma wall tiles but with the front face of the graphite heat shield still several centimeters outside the last closed plasma flux surface. However, due to the closer distance between the plasma and the first wall at the midplane, FILD2 has only been placed such that the front face of the graphite heat shield is $\sim 10 \mathrm{~mm}$ in the limiter shadow and the aperture is behind the front face of the plasma tiles. The result is that most ion orbits coming in at a small angle relative to the vessel wall will be blocked by the plasma facing wall tiles before reaching the aperture, as shown in Fig. 2(a). To resolve this issue, the local tiles were modified with a $7.8^{\circ}$ cut. The distance between the aperture and the scintillator is also reduced from $10 \mathrm{~mm}$ (for FILD1) to $5 \mathrm{~mm}$. Two thermocouples are internally routed and tacked against the wall of the probe tube to measure the temperature inside the FILD2 detector head. At the same time, these thermocouples serve as the bleed wire to prevent charge buildup.

In addition, a "D shape" probe head (Fig. 2(b)) based on the ASDEX design ${ }^{9}$ is adopted to enhance detection of ions

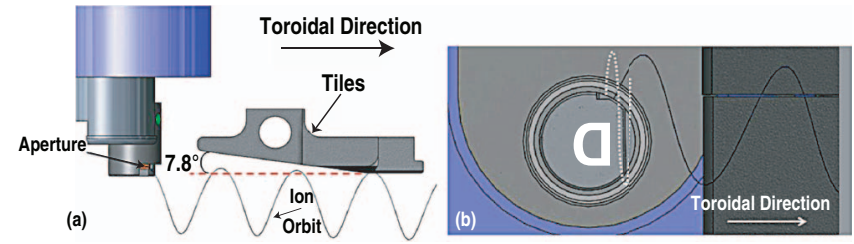

FIG. 2. Probe head and one side of local tile are viewed (a) from top looking down toward the tokamak midplane. A nearby tile is modified $\left(7.8^{\circ}\right.$ cut) for FILD2 to avoid blocking ion orbits (red dashed line shows the front surface of local tile before modification). (b) Radial viewed from plasma towards the vessel wall. The flipped "D-shaped" probe head of FILD2 avoids scrape-off of deeply trapped ions (white dashed orbit). with large pitch angles which might otherwise be blocked by the probe head. The detection range of a FILD is determined by the geometry of the probe head and aperture. Like FILD1, FILD2 is capable of detecting a wide range of lost ions with $35^{\circ}$ to $85^{\circ}$ in pitch angle $(\alpha)$ and 1.5 to $8.5 \mathrm{~cm}$ in gyroradius $\left(\mathrm{r}_{L}\right)$.

\section{BENEFITS FROM THE TWO FILDS}

On DIII-D, there are four pairs of neutral beams injectors located at $30^{\circ}, 150^{\circ}, 210^{\circ}, 330^{\circ}$ toroidal angles. The pair at $210^{\circ}$ is counter-injected relative to the normal plasma current direction while the other three pairs are co-injected. The two sources in each pair are labeled by their toroidal angle and position Left (L) or Right (R) within the beam housing, e.g. $330 \mathrm{~L}$ and $330 \mathrm{R}$. For the co-injection, the left beam is more tangential while the right beam is more tangential for counterinjection. Although all neutral beams produce prompt losses, both experiments and simulations show that prompt losses from beams other than the two counter-injected beams are barely connected to FILD $1 .{ }^{10}$ The addition of the midplane FILD2 has compensated for the lack of co-beam loss detection on FILD1. Prompt losses from several beam sources have been observed at FILD2. Dedicated plasmas for prompt loss study (calibration shots) are conducted by firing one neutral beam at a time during the plasma current ramp-up. Figure 3(a) shows a typical camera view during prompt loss events overlaid with the mapping grid. The camera is exposed for $0.5 \mathrm{~ms}$ (maximum exposure time for $160 \mathrm{fps}$ operation mode) starting from $494 \mathrm{~ms}$ during the $330 \mathrm{~L}$ beam pulse from $490-500 \mathrm{~ms}$. The grid is calculated using a revised version of the code NLSDETSIM (Ref. 11) which takes account of the magnetic field direction at the probe position and the geometry of the aperture and scintillator. The losses at $\alpha \sim 61^{\circ}$ and $r_{L}$ centered at $4.0 \mathrm{~cm}$ are due to prompt losses of the full energy component $(74.5 \mathrm{keV})$ of ions from the co-injected 330L neutral beam. Figure 3(b) shows the calculated maximum possible striking range on the scintillator by an ion which has $\mathrm{r}_{L}=4.0 \mathrm{~cm}$ and $\alpha \sim 61^{\circ}$ at the aperture. The resolution is a function of pitch angle and gyroradius. For
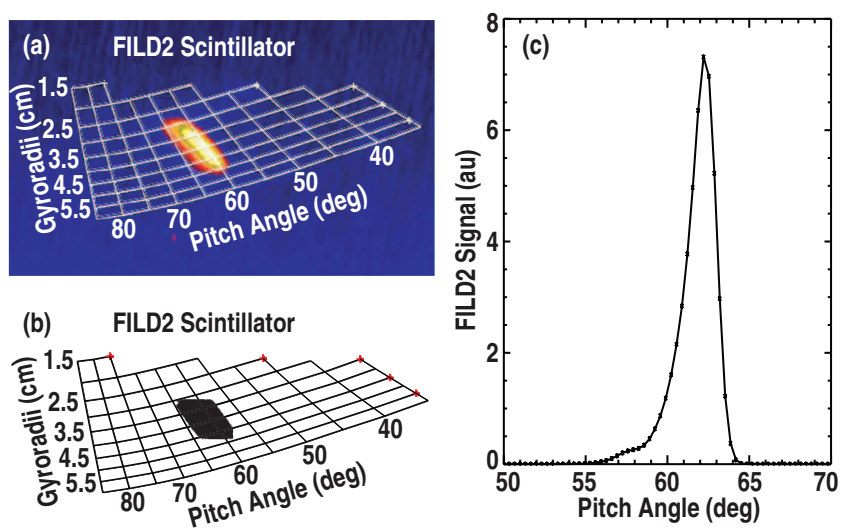

FIG. 3. (a) Camera frame with mapping grid overlaid for shot 146 103, 494 $\mathrm{ms}$, the bright strip is losses from the full energy component of the $330 \mathrm{~L}$ neutral beam; (b) the calculated maximum striking range on the scintillator for lost ions with $\mathrm{r}_{L}=4.0 \mathrm{~cm}$ and $\alpha \sim 61^{\circ}$ at the aperture; (c) the pitch angle resolution $\left( \pm 4^{\circ}\right.$ centers at $\left.\alpha \sim 62^{\circ}\right)$ calculated with synthetic modeling matches experimental observation well. 
the example shown in Fig. 3, the resolution is approximately $\pm 4^{\circ}$ in pitch angle and $\pm 1.3 \mathrm{~cm}$ in gyroradius. The pitch angle resolution is calculated using synthetic modeling ${ }^{10}$ which calculates the ion orbit by solving the Lorentz equation with given ion energy, pitch angle, and plasma magnetic equilibrium. The orbit is traced from detector back into the plasma and is identified as a possible detected orbit if it intersects with the neutral beam footprint with the proper velocity and position. The violet ion orbit in Fig. 1 is an example. The result shown in Fig. 3(c) is from an ion orbit calculation with experimentally observed energy $(74.5 \mathrm{keV})$ but a set of pitch angles within the full detectable range. The calculated pitch angles peak near $62^{\circ}$ with $\pm 4^{\circ}$ uncertainty which is similar to the experimental observation in Fig. 3(a). In experiments with multiple beams, this synthetic modeling code and/or the reverse-orbit tracing simulation are used to help determine the possible loss origins and type of ion orbits.

Having two FILDs at different poloidal locations allows for the exploration of fast ion losses in different plasma shapes. So far, the strongest loss signals collected at FILD1 are in oval shape plasmas while FILD2 is also sensitive to losses in circle and diverted shape plasmas (Fig. 1). The two poloidally separated FILDs allow for collecting fast ion losses while ion orbits change as the plasma current evolves (poloidal magnetic field changes). During the current ramp up, it is commonly observed on DIII-D that prompt losses at FILD2 occur at higher plasma currents while FILD1 observes prompt losses at lower plasma currents. This is because at lower plasma current, the large banana width orbits tend to hit poloidally below the midplane and miss FILD2. However, there is no simple critical plasma current value or banana orbit width due to the complexity of the ion orbits.

The process by which instabilities expel confined fast ions on different types of orbits can be studied with the two FILDs at different locations. For example, high amplitude of convective fast ion losses induced by Alfvén eigenmode activity is observed at the detector below the midplane. ${ }^{12} \mathrm{Sim}-$ ulation shows those ions are dominantly counter-going passing particles kicked by Alfvén eigenmodes onto banana lost orbits. ${ }^{6}$ Toroidally dependent Alfvén eigenmode-induced fast ion loss was observed on the midplane FILD and will be reported in a separate paper.

The combination of measurements at the two FILDs improved our ability to capture more information on the fast ion loss. Two examples are shown in Fig. 4. There are strong and continuous Alfvén eigenmodes during the current ramp-up phase of shot 146091 (left column) and shot 146082 (right column) as shown in Figs. 4(a) and 4(d)) magnetic data spectra. In shot 146 091, the 30L and 330L beams are alternatively on every $10 \mathrm{~ms} ; 150 \mathrm{~L}$ and $330 \mathrm{~L}$ beams are constantly firing. Thus, the total injection power is nearly constant except for a beam blip of 210R from 380 to $390 \mathrm{~ms}$. FILD2 detects coherent TAE loss starting from $350 \mathrm{~ms}$ (Fig. 4(b)) with strong signals during 350-370 ms and 400-440 ms. FILD1 detects weak coherent TAE and RSAE loss at 330-340 ms and TAE loss during the 210R beam blip (380-390 ms) (Fig. 4(c)). In shot 146 082, from 350 to $480 \mathrm{~ms}$, FILD2 detects strong coherent TAE loss (Fig. 4(e)) during every 330L beam blip while there is no detectable loss signal on FILD1 (Fig. 4(f)).
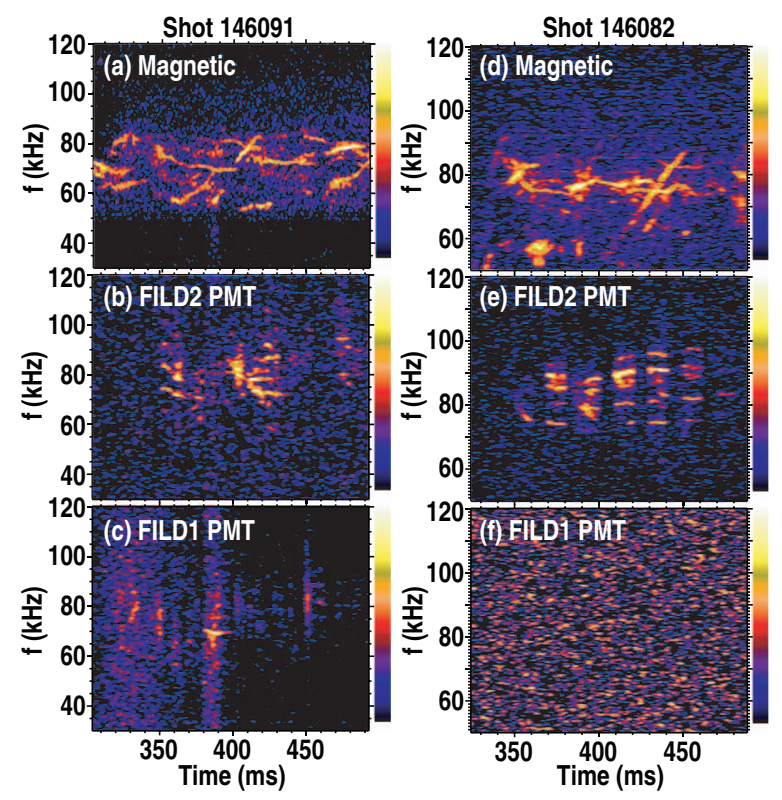

FIG. 4. Many TAEs (fixed frequency) and some RSAEs (frequency chirping) are observed in shot 146091 (left column) and shot 146082 (right column) by edge magnetic probes [(a) and (d)]. In shot 146 091, FILD2 detects coherent TAE loss (b) while FILD1 detects weak coherent TAE and RSAE loss near $330 \mathrm{~ms}$ and strong TAE loss at 380-390 ms and 460-470 ms (c) when counter-injected 210R was fired. In shot 146082, FILD2 observes strong coherent TAE loss every time window (e) when co-injected 330L was fired. However, there is no detectable coherent loss observed on FILD1 (f).

In summary, combined measurements with the new midplane FILD and the original FILD have increased the detection range of fast ion losses on DIII-D, broadened the measurement capability in terms of plasma shape and current profiles, and improved our understanding of fast ion loss processes.

\section{ACKNOWLEDGMENTS}

The authors are grateful to the DIII-D team for the engineering support and operating the machine. This work was supported in part by the DOE (Grant Nos. DE-FG0394ER54271, DE-FC02-04ER54698 and SC-G903402).

${ }^{1}$ W. W. Heidbrink and G. J. Sadler, Nucl. Fusion 34, 535 (1994).

${ }^{2}$ M. Garcia-Munoz et al., Nucl. Fusion 49, 085014 (2009).

${ }^{3}$ Y. B. Zhu, W. W. Heidbrink, and L. D. Pickering, Nucl. Fusion 50, 084024 (2010).

${ }^{4}$ W. W. Heidbrink et al., Plasma Phys. Controlled Fusion 53, 085028 (2011).

${ }^{5}$ R. K. Fisher, D. C. Pace, M. Garcia-Munoz, W. W. Heidbrink, C. M. Muscatello, M. A. Van Zeeland, and Y. B. Zhu, Rev. Sci. Instrum. 81, 10 D307 (2010).

${ }^{6}$ M. A. Van Zeeland et al., Phys. Plasmas 18, 056114 (2011).

${ }^{7}$ H. H. Duong, W. W. Heidbrink, E. J. Strait, T. W. Petrie, R. L. Lee, R. A. Moyer, and J. G. Watkins, Nucl. Fusion 33, 749 (1993).

${ }^{8}$ S. J. Zweben et al., Nucl. Fusion 35, 893 (1995).

${ }^{9}$ M. Garcia-Munoz, H.-U. Fahrbach, H. Zohm, and ASDEX Upgrade Team, Rev. Sci. Instrum. 80, 053503 (2009).

${ }^{10}$ D. C. Pace, R. K. Fisher, M. Garcia-Munoz, D. S. Darrow, W. W. Heidbrink, C. M. Muscatello, R. Nazikian, M. A. Van Zeeland, and Y. B. Zhu, Rev. Sci. Instrum. 81, 10D305 (2010).

${ }^{11}$ S. J. Zweben, R. L. Boivin, M. Diesso, S. Hayes, H. W. Hendel, H. Park, and J. D. Strachan, Nucl. Fusion 30, 1551 (1990).

${ }^{12}$ D. C. Pace, R. K. Fisher, M. Garcia-Munoz, W. W. Heidbrink, and M. A. Van Zeeland, Plasma Phys. Controlled Fusion 53, 062001 (2011). 